\title{
Preface: photosynthesis and hydrogen energy research for sustainability
}

\author{
Tatsuya Tomo $^{1} \cdot$ Suleyman I. Allakhverdiev ${ }^{2,3,4,5}$
}

Published online: 10 April 2017

(C) Springer Science+Business Media Dordrecht 2017

\begin{abstract}
Energy supply, climate change, and global food security are among the main chalenges facing humanity in the twenty-first century. Despite global energy demand is continuing to increase, the availability of low cost energy is decreasing. Together with the urgent problem of climate change due to $\mathrm{CO}_{2}$ release from the combustion of fossil fuels, there is a strong requirement of developing the clean and renewable energy system for the hydrogen production. Solar fuel, biofuel, and hydrogen energy production gained unlimited possibility and feasibility due to understanding of the detailed photosynthetic system structures. This special issue contains selected papers on photosynthetic and biomimetic hydrogen production presented at the International Conference "Photosynthesis Research for Sustainability-2016", that was held in Pushchino (Russia), during June 19-25, 2016, with the sponsorship of the International Society of Photosynthesis Research (ISPR) and of the International Association for Hydrogen Energy (IAHE).
\end{abstract}

Suleyman I. Allakhverdiev

suleyman.allakhverdiev@gmail.com

1 Department of Biology, Faculty of Science, Tokyo University of Science, Kagurazaka 1-3, Shinjuku-Ku, Tokyo 162-8601, Japan

2 Controlled Photobiosynthesis Laboratory, Institute of Plant Physiology, Russian Academy of Sciences, Botanicheskaya Street 35, Moscow 127276, Russia

3 Institute of Basic Biological Problems, Russian Academy of Sciences, Pushchino, Moscow Region 142290, Russia

4 Department of Plant Physiology, Faculty of Biology, M.V. Lomonosov Moscow State University, Leninskie Gory 1-12, Moscow 119991, Russia

5 Bionanotechnology Laboratory, Institute of Molecular Biology and Biotechnology, Azerbaijan National Academy of Sciences, Baku, Azerbaijan
This issue is intended to provide recent information on the photosynthetic and biohydrogen production to our readers.

Keywords Photosynthesis - Artificial photosynthesis · Water oxidation $\cdot$ Hydrogen energy $\cdot$ Renewable energy · International conference

"I believe that water will one day be used as a fuel, because the hydrogen and oxygen which constitute it, used separately or together, will furnish an inexhaustible source of heat and light. I, therefore, believe that, when coal deposits are oxidized, we will heat ourselves by means of water. Water is the coal of the future." Jules Verne, 1875.

More than 3 billion years ago, living organisms developed the ability to capture solar energy efficiently for producing organic molecules by photosynthesis. The photosynthetic process allowed high life diversity, as witnessed by the fossil records. Indeed, over eons of time photosynthesis has provided the oil, gas and coal for us to power our technologies, heat our homes and produce the wide range of chemicals and materials for everyday life.

It is obvious that fossil fuels (i.e., petroleum, natural gas and coal) while being rapidly depleted meet most of the world's energy demand today. Their utilization, however, causes global warming, climate change, ozone layer depletion, acid rain, and pollution, all of which are pose great dangers for the environment of our planet Earth. Many engineers and scientists came to the conclusion that the replacement of the fossil fuel system by the Hydrogen Energy System could be the solution of these global problems. Hydrogen is the most efficient and the cleanest fuel. Its combustion does not produce greenhouse gases, ozone layer depleting chemicals, acid rain ingredients, and finally environmental pollution. Being a renewable energy carrier, hydrogen has a high specific 
heat of combustion. It is a clean fuel with zero carbon emission. It is possible to use hydrogen in internal combustion engines to generate mechanical power or in fuel cells to generate electricity. As hydrogen can be produced from many natural sources, it is expected to have a stable price in the future, independent of the fluctuation in price and availability of single sources. Hydrogen is a synthetic fuel and it should be manufactured.

There are various methods to manufacture hydrogen: direct thermal, thermochemical, electrochemical, biological methods, etc. Among these, biological methods have the potential of providing the most cost effective hydrogen. Due to this fact, many research groups around the world work on biological hydrogen production. Nowadays, it is important to develop renewable and clean energy sources for the future. In this regard, photosynthesis provides a successful example of solar energy convertion into fuel with electrons extracted from water by means of light as the only energy source. It would be wise to look at photosynthesis in more detail, because this processes contains many clues for us to learn. An economy and transport infrastructure based on molecular hydrogen and fuel cells could decrease our dependence on oil and the concomitant environmental consequences. Positively affecting the energy security, such approach would mitigate air pollution and global climate change. Moreover, biological production of hydrogen by photosynthesis may become a valuable alternative to chemical and electrochemical technologies. Firstly, solar energy and water are renewable energy sources, being rather cheap. Secondly, burning of $\mathrm{H}_{2}$ is clean renewable process, with the release of water as end product. All biological solardriven methods of $\mathrm{H}_{2}$ production in green algae, cyanobacteria and higher plants are based on photosynthesis.

Human life existed in past and exists today due to the photosynthesis. We should be grateful to all the plants, algae and cyanobacteria for supplying us with oxygen, food, biomass, and bioenergy. As we know, the present life depends on oxygenic photosynthesis. It provides the air for living organisms to breath, and photosystem II can derive an unlimited source of electrons from water for oxygen and hydrogen using solar energy.

Today we have considerable knowledge of the workings of photosynthesis and its photosystems, including the water oxidation reaction. However, many questions and details remain unanswered. Full understanding of the photosynthetic process is not only a satisfying intellectual pursuit, but is also an important goal as we strive to improve agricultural yields and develop new solar technologies for splitting of water into pure $\mathrm{O}_{2}$ and $\mathrm{H}_{2}$ for use as a potential fuel source, as Jules Verne predicted 142 years ago.

We are pleased to bring out this special issue. This issue contains selected and invited papers presented at the International Conference in Pushchino (Russia).
The International conference "Photosynthesis and Hydrogen Energy Research for Sustainability-2016: in honor of Nathan Nelson and Nejat Veziroglu" is the seventh in series of already traditional conferences "Photosynthesis Research for Sustainability" held in Canada (2004), Russia (2007, 2014), Azerbaijan (2011, 2013), and Greece (2015) (see Allakhverdiev et al. 2012, 2013, 2014, 2016). Nearly 170 participants from 22 countries visited Institute of Basic Biological Problems RAS, Pushchino, the host of the conference. This conference was held during June 19-25, 2016 and was a great chance for discussions of molecular and global aspects of photosynthesic research and biohydrogen production (for more details see http://photosynthesis2016. cellreg.org/). The scientific program was quite exciting; it covered the breadth and depth of photosynthesis and biohydrogen production. This international conference covered almost all the important aspects of photosynthesis, and their relationship to global issues, as well as hydrogen production and artificial photosynthesis. In this meeting, work performed around the world was presented that focused not only on the understanding of the fundamental aspects of natural photosynthesis, but also on how it impacts research and development of new technologies for food and fuel production. All the selected and invited lectures provided in-depth analyses of recent progress in the studies of photosynthesis for sustainability. In addition, this conference was an important venue for international students, postdocs and other young investigators to expand their knowledge and understanding of the entire field of photosynthesis. At this conference, awards were given to 16 young investigators. We sincerely hope this meeting was enjoyable, fruitful, and memorable for all participants (see Tsygankov et al. 2017; Hou et al. 2017). We wish to express our cordial salutations to all of you.

We invite the readers to the next conference on 8th International Conference "Photosynthesis and Hydrogen Energy Research for Sustainability-2017" in which will honor A.S. Raghavendra (of University of Hyderabad), William Cramer (of Purdue University) and Govindjee (of University of Illinois at Urbana-Champaign); it will be held during the Fall of 2017 (from October 30 to November 4), at the University of Hyderabad, Hyderabad, India (See https:// prs.science).

Finally, we would like to deeply thank all the authors for their outstanding contributions, all the reviewers for their constructive and informative comments to improve the quality of the manuscripts, and all the conference attendees for stimulating discussions. We greatly acknowledge Terry M. Bricker (Editor-in-Chief) for his support and assistance. We are very grateful to Ellen Klink and Jaap van der Linden of Springer for their help during the production of this special issue. We thank the ISPR and the IAHE for supporting the conference. Finally, we appreciate the financial 
support from the Russian Science Foundation (Grant \#1514-30007 to SIA).

\section{References}

Allakhverdiev SI, Huseynova IM, Govindjee (2012) International conference on "Photosynthesis research for sustainability-2011, July 24-30, 2011, Baku, Azerbaijan. Photosynth Res 110:205-212

Allakhverdiev SI, Huseynova IM, Govindjee (2013) International conference on 'Photosynthesis research for sustainability-2013: in honor of Jalal A. Aliyev', held during June 5-9, 2013, Baku, Azerbaijan. Photosynth Res 118:297-307

Allakhverdiev SI, Tomo T, Govindjee (2014) International conference on "Photosynthesis research for sustainability-2014: in honor of
Vladimir A. Shuvalov', held on June 2-7, 2014, in Pushchino, Russia. Photosynth Res 122:337-347

Allakhverdiev SI, Tomo T, Stamatakis K, Govindjee (2016) International conference on "Photosynthesis research for sustainability-2015: in honor of George C. Papageorgiou', September 21-26, 2015 Crete, Greece. Photosynth Res 130:1-10

Hou HJM, Tomo T, Allakhverdiev SI (2017) An introduction to the special issue section on "The 7th International Conference on Photosynthesis and Hydrogen Energy Production in Honor of Nathan Nelson and T. Nejat Veziroglu, 19-25 June 2016, Pushchino, Russia”. Intl J Hydrog Energy. doi:10.1016/j. ijhydene.2016.11.052

Tsygankov AA, Allakhverdiev SI, Tomo T, Govindjee (2017) International conference on "Photosynthesis research for sustainability-2016" in honor of nathan nelson and turhan nejat veziroglu. Photosynth Res 131(2):227-236 